\title{
Calidad de vida en pacientes con osteoporosis en atención primaria
}

\author{
Mercedes Sánchez Barba \\ Departamento de Estadística, Universidad de Salamanca, España.
}

Dirección postal: Centro de Salud "Doctor Alfonso Sánchez Montero", Calle Alfareros, n 4

37007 Salamanca (España)

Ce: mersanbar@usal.es

\author{
Ma. PurificaciónVicente Galindo* Ana Martin Casado*** \\ Enrique Vicente Rivera * Jose Luis Vicente Villardón***
}

\section{Ma. Purificación Galindo-Villardón}

Resumen. El objetivo de esta investigación fue evaluar la calidad de vida en pacientes clasificados como osteoporóticos en el Ámbito de la Atención primaria. Se llevó a cabo un diseño observacional transversal, los pacientes fueron reclutados de forma consecutiva en un Centro de Salud de Atención primaria de Salamanca (España). Se utilizó un cuestionario sociodemográfico, el EuroQol-5D, para evaluar calidad de vida relacionada con la salud y el QUALEFFO para evaluar calidad de vida en pacientes clasificados como osteoporóticos y que no ha sido aplicado en Atención primaria. En los resultados para las mujeres, se observaron diferencias significativas entre pacientes clasificados como osteoporóticos y no osteoporóticos en todas las dimensiones de ambos cuestionarios. Para los hombres esas diferencias fueron significativas solo para ciertas dimensiones. Los resultados reflejan que la calidad de vida de los pacientes clasificados como osteoporóticos es más baja que para los no osteoporóticos, en todas las dimensiones de la calidad de vida evaluadas.

Palabras clave: Calidad de Vida, Osteoporosis, Qualeffo, EuroQol-5D, QUS

Abstract. Objective: Evaluate quality of life in osteoporotic patients in the Field of Primary Care. Method. The study design is observational, where patients were recruited consecutively in a Primary Care Center of Salamanca (Spain). To get information we used a socio demographic questionnaire. The EuroQol-5D, questionnaire designed to assess quality of life related to health and QUALEFFO questionnaire specifically designed to assess patients with osteoporosis that had not been applied in the field of Primary Care. Results: For the group of women, the differences found between the osteoporotic, and no osteoporotic women were statistically significant, for all dimensions of both questionnaires. However, for the group of men these differences were significant only to certain dimensions. Conclusions: The results indicate that the quality of life of osteoporotic patients is worse that of no osteoporotic patients. This holds for all the quality of life dimensions analysed.

Key Words: Quality of Life, osteoporosis, Qualeffo, EuroQol-5D, QUS

*Facultad de medicina, Universidad de Salamanca, España

**Centro de Salud “Alfonso Sánchez Montero", Salamanca, España

***Departamento de Estadística, Universidad de Salamanca, España 
Sánchez, Vicente, Martin, Vicente, Vicente y Galindo

\section{Introducción}

La osteoporosis es una enfermedad sistémica del esqueleto, caracterizada por la pérdida de masa ósea, con pérdida de la arquitectura normal del hueso y aumento de la fragilidad (Consensus Development Conference, 1993).

La Organización Mundial de la Salud (OMS) en 1993 definió la osteoporosis como una enfermedad metabólica sistémica caracterizada por la disminución de la masa ósea y una alteración de la microarquitectura del hueso que aumenta la fragilidad del hueso y conlleva a un incremento del riesgo de fractura. Esta definición se mantiene en la revisión de la guía europea de osteoporosis de 2008. La osteoporosis afecta a un gran número de personas mayores debido a que la masa ósea disminuye después de la menopausia, en las mujeres, y con el incremento de la edad, en ambos sexos. Se estima que el $30-40 \%$ de las personas mayores de 60 años tienen probablemente osteoporosis y alrededor del 66\% algún grado de osteopenia (Orozco, Montero \& Prieto, 2009). La fractura osteoporótica presenta un impacto sanitario y económico de gran magnitud en las sociedades actuales de los países desarrollados, ya que por una parte existe un déficit de la calidad de vida relacionada con la salud y por otra parte también existe un elevado coste económico que ocasiona una sobrecarga importante para cualquier sistema de salud.

En España, según estimaciones directas, los costes generados desde el primer transporte al hospital, hasta que se pasa la primera revisión viene a ser de unos 4000 a 5000 euros por paciente lo que significa que en la actualidad los gastos totales por años puedan estar comprendidos entre 350 a 300 millones de euros (Abidanza, 2005).

La osteoporosis en el hombre es un fenómeno poco analizado, aunque la pérdida de masa ósea en relación con la edad es común tanto en hombres como en mujeres. Según la International Osteoporosis Foundation (2006), 1 de cada 3 mujeres y 1 de cada 5 varones de edad mayor a los 50 años sufrirá una fractura relacionada con la osteoporosis. En la actualidad la osteoporosis que afecta al sexo masculino está adquiriendo mayor importancia (García, García, Mateo y Holgado, 2008). No existen criterios diagnósticos establecidos para la osteoporosis en el hombre, aunque sus características son diferentes de las de la mujer. Aunque no existe paralelismo con la menopausia de la mujer, los estudios epidemiológicos realizados en Estados Unidos, Alemania y Argentina indican que el contenido mineral óseo en el hombre comienza a descender a partir de los 50 años, y se acelera después de la sexta década. Concomitantemente en el varón se observa un aumento progresivo de fracturas, sobre todo de cadera. 
La mortalidad por fractura de cadera en el hombre con osteoporosis es el doble que en la mujer. En hombres con edades comprendidas entre los 55 y los 90 años se produce una reducción de las hormonas sexuales que intervienen en el remodelado óseo, o que ciertas proteínas de las hormonas sexuales se pueden convertir en factor predictivo de la pérdida de masa ósea (Sosa y Gómez de Tejada, 2008)

Se calcula que en España existen cerca de dos millones de mujeres con osteoporosis frente a 600.000 hombres; además se prevé que en el año 2050 la incidencia de las fracturas se multiplicará por 10 en varones.

Entre los factores de riesgo podemos destacar: sexo femenino, menopausia, raza blanca o asiática, sendentarismo, baja ingesta de calcio, deficiencia de vitamina $\mathrm{D}$, tabaquismo, alcoholismo, antecedentes familiares de osteoporosis, constitución física delgada, irregularidad menstrual, ciertas enfermedades como artritis reumatoide o la anorexia, ciertos medicamentos: cortisona, algunos anticonvulsionantes, etc. (Ortega, 2005).

Entre las causas de osteoporosis en el varón destaca el consumo de alcohol, el tratamiento de glucocorticoides y el hipogonadismo. La incidencia de fracturas vertebrales aumenta con la edad en ambos sexos; es mayor en mujeres de edad avanzada y en varones jóvenes debido a los traumatismos previos.

Existen numerosas pruebas bioquímicas que se determinan en análisis rutinarios que estudian el estado metabólico del hueso y que tienen su utilidad. Sin embargo el diagnóstico y detección de la pérdida de densidad ósea se realiza de forma indirecta a través de métodos indirectos radiológicos, ya que el estudio directo histológico de una muestra del tejido óseo a través de la biopsia queda fuera de la práctica rutinaria. Los métodos radiológicos son muy diversos, pero se pueden resumir en la utilidad de las radiografías convencionales de huesos y en la densitometría ósea.

La radiografía convencional tiene una baja sensibilidad en la detección de este proceso, ya que es necesaria una pérdida de al menos un 30\% de la masa ósea para detectar la osteoporosis en ciertos huesos.

Gracias a la densitometría ósea, tenemos capacidad para evaluar el esqueleto periférico, el esqueleto central y axial y el esqueleto total, así como los huesos trabeculares y corticales con un alto grado de precisión y sensibilidad.

En atención primaria se ha impuesto la utilización de los ultrasonidos periféricos (calcáneo, metacarpo, radio, etc.). Los sistemas por ultrasonidos, conocidos como QUS (Quantitative Ultrasound), son técnicas que se basan en la emisión de ondas de alta frecuencia a través de un hueso periférico. El modelo más ampliamente estudiado es el que se emplea para determinar la masa ósea del calcáneo, ya que tiene una composición porosa o trabecular en un $95 \%$ y también gracias a su morfología, pues se trata de un hueso plano y simétrico, de fácil acceso y que permite la localización precisa y exacta del 
Sánchez, Vicente, Martin, Vicente, Vicente y Galindo

transductor en medidas repetidas. Se trata de una técnica que necesita poca instalación, más barata ( $\mathrm{y}$, por tanto, puede ofrecer más accesibilidad que las técnicas ionizantes), que no irradia, y que necesita poco tiempo de exploración (unos 5 minutos). La prueba consiste en poner la zona a medir en contacto con dos transductores (uno a cada lado). Según el modelo de conducción de los ultrasonidos existen dos tipos de modelos: los que utilizan la técnica en agua (la región de exploración se sumerge en agua y los transductores se sitúan a ambos lados de la cubeta que contiene el agua) y los de la técnica seca (utilizan un gel sobre el que se aplican los transductores) (Orozco et als., 2009).

He, Fan, Hans, Li, Wu y Njeh (2000) demostraron que los resultados obtenidos por QUS representan correlaciones significativas con la Densidad Mineral Ósea (DMO) obtenida con la densitometría DEXA (Dual Energy Xray Absorptimetry). Aunque no podemos aplicar los criterios de la OMS, definidos específicamente para la DEXA, a la QUS, debido a que son medidas de distintos aspectos del hueso, que se comporta de distinta forma ante medios físicos diferentes (rayos X y ultrasonidos) (Frost, Blake \& Fogelman, 2000; Gómez de Tejada \& Sosa, 2002). No obstante, el hecho de que no sea equiparable a la DEXA no invalida la QUS como método de valoración de la osteoporosis (Sosa y Gómez de Tejada, 2006). En nuestra población disponemos de unos valores de normalidad para el QUS de calcáneo de ambos sexos, de la marca Sahara, realizados por el Grupo de Investigación en Ultrasonidos y Metabolismo Óseo (GIUNO), que considera osteoporosis un valor de $t$-score $<1.8 \mathrm{DE}$, por lo que en este caso no consideramos que hay riesgo de osteoporosis (Orozco et al, 2009).

Además, Manuel Sosa Henríquez, coordinador de la IV Reunión Nacional del Grupo de Trabajo en osteoporosis de la Sociedad Española de Medicina Interna (SEMI), celebrada en Barcelona en abril de 2008 a la que asistieron unos 150 expertos nacionales y referentes internacionales, explicó que las técnicas de ultrasonidos están demostrando que pueden contribuir notablemente a la estimación del riesgo de fractura por osteoporosis y a la valoración de la estructura del hueso.

Los sistemas QUS permiten obtener parámetros determinados como la atenuación del ultrasonido (Broadband Ultrasound Attenuation, BUA) que valora la densidad y estructura ósea a partir de la atenuación de la señal ultrasónica al atravesar el calcáneo y la velocidad del ultrasonido (Velocity or Speed of Sound, VOS o SOS) que se relaciona con la elasticidad y densidad del tejido óseo. BUA y VOS se comparan con los resultados obtenidos de una población normal, calculando el Z-Score, que mide el número de desviaciones estándar que el valor BUA se desvía del valor medio de la población, para una edad y sexo particular: 
Calidad de vida en pacientes con osteoporosis en atención primaria

$$
\text { puntuación } \quad \mathrm{z}=\frac{\text { BUA sujeto }- \text { BUA medio para su edad y sexo }}{\text { Desviación estándar de su grupo de edad y sexo }}
$$

y el T-Score, similar al Z-Score pero referido al pico de masa ósea:

$$
\text { puntuación } \mathrm{t}=\frac{\text { BUA sujeto }- \text { BUA"pico de masa ósea" }}{\text { Desviación estándar del"pico de masa ósea" }}
$$

En el contexto clínico, la calidad de vida está relacionada con los aspectos que se ven afectados por una enfermedad o su tratamiento. Según Alonso (2002) la Calidad de Vida Relacionada con la Salud (CVRS) es el valor asignado a la duración de la vida, modificado por el deterioro, estados funcionales, las percepciones y las oportunidades sociales que se ven influidas por una enfermedad, lesión o tratamiento.

La definición de la calidad de vida es compleja y controvertida. La definición que se debe tener en cuenta en estudios sobre calidad de vida debe centrarse en el impacto de la salud de una persona, en su capacidad para llevar una vida plena después del tratamiento (Velarde-Jurado \& ÁvilaFigueroa, 2002).

"Percepción de la calidad de vida" será entendido como un constructo hipotético (no directamente medible), sinónimo de bienestar subjetivo, teniendo en cuenta sus diferentes ámbitos: bienestar físico, emocional y social. El grado de bienestar experimentado por el paciente depende de: su salud objetiva, de cómo el paciente la percibe, de su satisfacción en el entorno y de las características del individuo. La osteoporosis puede afectar negativamente a la calidad de vida, reduciendo el funcionamiento físico y la movilidad, y afectando las actividades de la vida diaria. También se produce a menudo un estado de ánimo bajo, depresión y aislamiento social. Se han llevado a cabo varios estudios (Randell, Nguyen, Bhalerao, Silverman, Sambrook, Eisman, 2000), que comparan un grupo control con pacientes que habían sufrido fracturas de cadera, demostrando que los pacientes con fractura de cadera vieron reducida su calidad de vida, principalmente en las dimensiones física y social. En un ensayo transversal (Olesksik et al, 2000) en el que analizó el estado de salud en las mujeres postmenopáusicas con baja densidad mineral ósea, con o sin fracturas de vértebra, los resultados indicaron que los pacientes con fractura de vértebra presentan peor calidad de vida que los pacientes sin fractura. Además, la asociación entre las fracturas de vértebra y la calidad de vida depende de la localización de la fractura. También en varios ensayos en el análisis de la calidad de vida en pacientes con fractura de muñeca (Dolan, Torgenson y Kakarlapudi, 1999) se 
puso de manifiesto que este tipo de fractura también reduce la calidad de vida.

Dada la trascendencia social y económica que tiene la osteoporosis, y su incidencia cada vez mayor, también en hombres, está claro que la responsabilidad del diagnóstico, evaluación, tratamiento y, sobre todo, prevención global de la osteoporosis deberán orientarse rápidamente desde los especialistas a los médicos de atención primaria (Díaz, 2006), por lo que en este trabajo pretendemos conocer si la calidad de vida percibida por los pacientes clasificados como osteoporóticos evaluados en atención primaria, es más baja que en los sujetos clasificados como no osteoporóticos.

En la mayoría de los trabajos de CVRS, la definición aceptada es la asociada al instrumento de medida (cuestionario) utilizado. Por esta razón, el instrumento juega un papel crucial en investigaciones de CVRS. Para la evaluación de estos pacientes se puede utilizar instrumentos genéricos de CVRS. Estos cuestionarios son aplicables a cualquier tipo de población y enfermedad y permiten la comparación entre sujetos que presentan diversas patologías; sin embargo, no exploran en profundidad aspectos específicos de la osteoporosis (Lizán, Badia, 2003), en nuestro caso hemos utilizado el QUALEFFO (Questionnaire of the European Foundation for Osteoporosis) elaborado por Lips, Cooper, Agnus, Caulin, Johnell y Kanis (1999), un cuestionario específico para evaluar calidad de vida en pacientes con osteoporosis en ambiente hospitalario y que nunca ha sido aplicado para evaluar calidad de vida en atención primaria. Sánchez (2008), probó que el QUALEFFO sigue siendo válido al ser aplicado en screening en pacientes ambulatorios.

\section{Método}

\section{Selección de la muestra}

Se efectuó un estudio observacional, transversal, en el centro de Salud "Doctor Alfonso Sánchez Montero" de Salamanca. Se llevó a cabo un muestreo no probabilístico, consecutivo, siguiendo los siguientes criterios de inclusión: a) tener dos o más factores de riesgo elevado o bien, b) tener cuatro o más factores de riesgo moderado, o c) tener un factor de riesgo elevado y dos o más factores de riesgo moderado. La recogida de datos se llevó a cabo desde octubre de 2007 hasta octubre de 2008. Se explicó a cada paciente el objetivo del estudio, solicitando su participación en el periodo que le fue cedido el aparato de ultrasonidos al Centro de Salud y con el que se consiguió una muestra de 741 pacientes. No hubo ningún paciente que rechazase participar. 
A estos pacientes se les realizó una medición ultrasónica en el calcáneo, obteniéndose: el BUA y el T-Score. Este último valor permitió clasificar a los pacientes estudiados en sujetos con osteoporosis, con osteopenia y normales ${ }^{1}$. Los puntos de corte empleados en dicha clasificación fueron los siguientes: para puntuaciones de $T$ superiores a $-1.0 \mathrm{DE}$ el paciente era diagnosticado como sano, puntuaciones entre $-1 \mathrm{DE}$ y $-1.8 \mathrm{DE}$ pacientes con osteopenia y puntuaciones superiores $-1.8 \mathrm{DE}$ pacientes con osteoporosis, valores indicados en el densitómetro CUBA Clinical ${ }^{\mathrm{TM}}$ portátil, proporcionado por el Grupo Vita y cuyo sistema de medida fue validado por FDA (Food and Drugs Administrator).

Para evaluar la calidad de vida se utilizó un instrumento genérico, el EuroQol-5D y un instrumento específico para pacientes con osteoporosis (QUALEFFO), además se hicieron cinco preguntas para recoger datos sociodemográficos y laborales y 11 preguntas más para obtener datos de interés clínico. Las repuestas de los participantes a los cuestionarios se introdujeron en una base de datos y se analizaron con el programa estadístico SPSS, versión 16.0. Se calcularon estadísticos descriptivos de frecuencia de las variables cuantitativas estudiadas (media y desviación estándar [DE]). La asociación entre las variables se estableció mediante las pruebas de la $t$ de Student y la ji cuadrado, en dependencia del tipo de variable. El nivel de significación estadística empleado fue de $p<0.05$.

\section{Cuestionario EUROQOL-5D}

El cuestionario EUROQOL (EQ-5D), fue desarrollado en el marco europeo común que comenzó en 1987, iniciado entre otros, por el profesor Alan Williams en la Universidad de York en Inglaterra. En 1992, se adaptó el instrumento para su uso en España. Se realizó una prueba piloto con 10 pacientes y 10 individuos aparentemente sanos que sugirió algún cambio y mostró que la traducción era satisfactoria. Se evaluó la validez de concepto mediante un análisis factorial que mostró tres factores: "físico", "dolor" y "salud mental" (Rué \& Badía, 1996). Los resultados muestran que, en todos los casos, el EuroQol es capaz de discriminar entre mala salud y buena salud según las hipótesis previamente establecidas. Se realizó un estudio administrando el cuestionario en dos ocasiones separadas por un intervalo de 15 días, para comprobar la fiabilidad test-retest obteniendo coeficientes de correlación de Spearman altos, moderados y bajos en distintos estados de

\footnotetext{
${ }^{1}$ Entenderemos por normales aquellos pacientes para los que la densitometría por ultrasonidos ha dado valores considerados normales.
} 
Sánchez, Vicente, Martin, Vicente, Vicente y Galindo

salud (Alegre \& Nualart, 1995). Este cuestionario analiza dos tipos de información:

a) El sistema descriptivo que da información en relación a cinco dimensiones relativas a movilidad, cuidado personal, actividades cotidianas, dolor/malestar y ansiedad/depresión, codificándose cada una de ellas con un 1 cuando no se perciben problemas, con un 2 cuando se estiman algunos/moderados problemas y con un 3 cuando los problemas son muchos. El individuo debe marcar el nivel de problemas, dentro de cada dimensión, que mejor describe su estado de salud "en el día de hoy", generándose así, un total de 243 estados de salud teóricamente posibles. De este modo, se define el estado de salud del individuo mediante un número de cinco dígitos. El mejor estado de salud se describe 11111 y el peor 33333.

b) La Escala Visual Analógica (EVA), que va del 0 al 100; en la cual, se marca con un 100 el mejor estado de salud y con un " 0 " el peor estado de salud que pueda imaginarse, al aplicarse el día de la prueba. El individuo debe señalar en la escala el punto que, en su opinión, indica su estado de salud en el día de hoy. El valor asignado en la EVA puede utilizarse como un indicador cuantitativo del estado de salud de un individuo o de un grupo de individuos.

\section{Cuestionario QUALEFFO}

El cuestionario QUALEFFO fue validado en un estudio multicéntrico llevado a cabo en siete países (Lips et als, 1999) sobre pacientes de 55-80 años con osteoporosis clínica. La fiabilidad test-retest fue buena (0.54-0.90) y la consistencia interna de cada una de las cinco dimensiones fue alta (alfa de Cronbach, 0.80). Las puntuaciones medias del instrumento fueron significativamente más altas en pacientes con fracturas vertebrales que en los controles, en todas las dimensiones. Este instrumento ha sido recientemente adaptado para su uso en España (Badía \& Hermand, 1999), es el cuestionario más utilizado en medicina contemporánea para medir la calidad de vida en pacientes con osteoporosis, consta de 35 ítems, los cuales se refieren a siete dimensiones de salud: Dolor (cinco ítems), Actividades Cotidianas (tres ítems), Tareas Domésticas (cinco ítems), Movilidad (seis ítems), Actividades Sociales y de Tiempo Libre (cuatro ítems), Percepción de la Salud General (dos ítems) y Estado de Ánimo (10 ítems). La respuesta a cada ítem está también en escala ordinal, desde 1 (ningún problema) a 5 (muchos problemas).

Cada uno de los ítems tiene cinco posibles respuestas, dependiendo de la respuesta elegida el paciente recibe una puntuación en el ítem. La puntuación de cada dimensión se obtiene sumando los valores de respuesta de cada ítem de la dimensión y dividiendo este valor por el total de ítems respondidos de la 
dimensión. La puntuación total oscila de 1 a 5 , de modo que cuanta más alta es la puntuación peor es la calidad de vida, y se obtiene sumando la puntuación en cada dimensión y dividiéndola entre el número de dimensiones del cuestionario.

\section{Resultados}

De los 741 analizados, 550 (74.2\%) eran mujeres y 191 (25.8\%) hombres, con edades comprendidas entre los 40 y 70 años para ambos sexos; un 70\% de los pacientes están casados. Con los puntos de corte establecidos, la muestra quedó clasificada de la siguiente manera: 298 pacientes fueron clasificados como pacientes con osteoporosis, 229 con osteopenia y 192 como pacientes que no padecían la enfermedad (Figura 1).

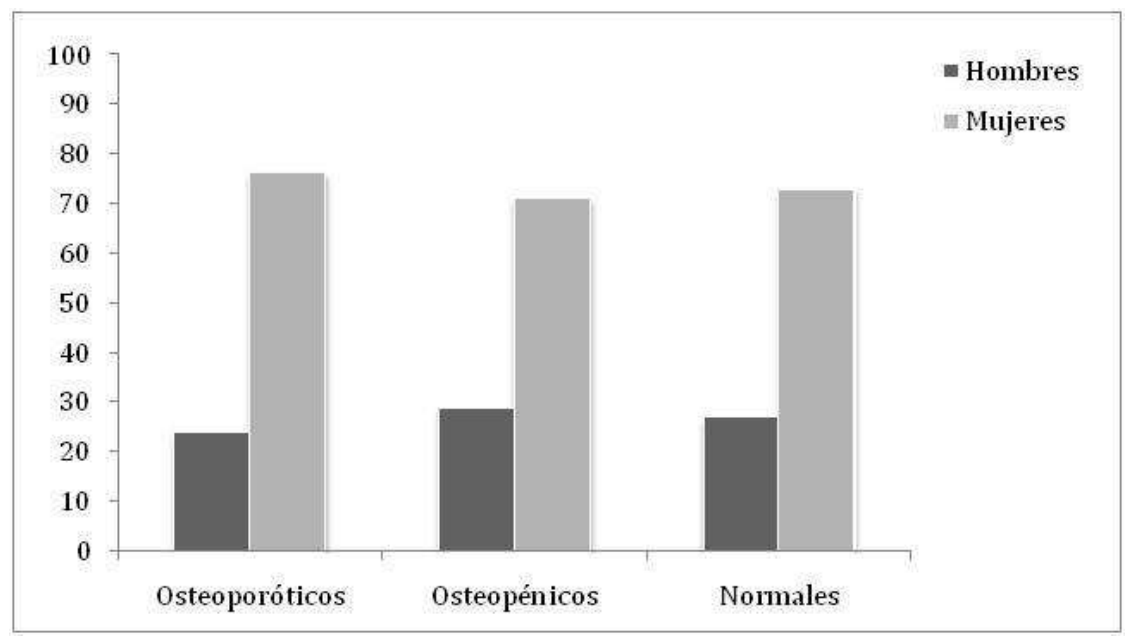

Figura.1. Distribución de frecuencias de la variable sexo segmentada por clasificación en relación a la osteoporosis.

Más de la mitad de los individuos de los pacientes clasificados como osteoporóticos tienen más de 60 años $(47.9 \%$ de los clasificados como no osteoporóticos); un $16.1 \% \quad(22.3 \%$ para los clasificados como no osteoporóticos) tienen entre 51 y 60 años; un 6.6\% (18.6\% de los clasificados como no osteoporóticos) entre 41 y 50 años y un $1.7 \%$ (11.2\% para los clasificados como no osteoporóticos) menos de 40 años. Estos porcentajes se mantienen para el grupo de los hombres y el grupo de las mujeres.

En cuanto a los datos de interés clínico, se encontró que: un 18.37\% de los pacientes considerados habían tenido fractura después de los 40 años y un $9.34 \%$ tienen antecedentes familiares con fractura de cadera. El 26.20\% de las 540 pacientes que contestaron a esta pregunta, tuvo menopausia prematura; el 
$54.94 \%$ tenía artrosis; un $7.48 \%$ tenía artritis; un 11.15\% (de 260 pacientes) tiene otras enfermedades reumáticas y el $40 \%$ (de 475 pacientes) manifiesta que es el problema de los huesos el que más influye en su estado de salud.

Evaluación de la calidad de vida con el cuestionario EUROQOL-5D

Al comparar la calidad de vida de los pacientes clasificados con osteoporosis y el resto hemos encontrado diferencias estadísticamente significativas para todas las escalas, en el grupo de las mujeres; todas con $p<$ 0.001 (Tabla 1).

Tabla 1.

Distribución porcentual para las cinco dimensiones del EuroQol, comparando mujeres clasificadas como osteoporóticas y como no osteoporóticas.

\begin{tabular}{lccc}
\hline & Nivel 1 & $\begin{array}{c}\text { Mujeres clasificadas } \\
\text { como osteoporóticas } \\
(\mathrm{n}=227)\end{array}$ & $\begin{array}{c}\text { Mujeres clasificadas } \\
\text { como no } \\
\text { osteoporóticas } \\
(\mathrm{n}=302)\end{array}$ \\
\hline Movilidad* $\mathrm{n}(\%)$ & $115(50.7)$ & $196(64.9)$ \\
& Nivel 2 & $109(48.0)$ & $105(34.8)$ \\
Cuidadol 3 & $3(1.3)$ & $1(0.3)$ \\
& Nivel 1 & $176(77.5)$ & $265(87.7)$ \\
& Nivel 2 & $43(18.9)$ & $36(11.9)$ \\
Actividades Cotidianas* $\mathrm{n}(\%)$ & Nivel 3 & $8(3.5)$ & $1(0.3)$ \\
& Nivel 1 & $129(56.8)$ & $231(76.5)$ \\
& Nivel 2 & $84(37.0)$ & $68(22.5)$ \\
Dolor/Malestar* $\mathrm{n}(\%)$ & Nivel 3 & $14(6.2)$ & $3(1)$ \\
& Nivel 1 & $38(16.7)$ & $109(36.1)$ \\
& Nivel 2 & $135(59.6)$ & $145(48.0)$ \\
Ansiedad/Depresión* $\mathrm{n}(\%)$ & Nivel 3 & $54(23.8)$ & $48(15.9)$ \\
& Nivel 1 & $107(47.1)$ & $195(64.6)$ \\
& Nivel 2 & $88(38.8)$ & $88(29.1)$ \\
& Nivel 3 & $32(14.1)$ & $19(6.3)$ \\
\hline
\end{tabular}

Nota. $* \mathrm{p}<0.05$

Sin embargo para el grupo de los hombres hemos encontrado resultados estadísticamente significativos solamente en las escalas de Movilidad ( $\mathrm{p}=$ 0.004) y Actividades Cotidianas $(p=0.002)$ (Tabla 2) y no en las escalas Cuidado Personal, Dolor/Malestar y Ansiedad/Depresión ( $\mathrm{p}>0.05)$. 
Tabla 2.

Distribución porcentual para las cinco dimensiones del EuroQol, comparando hombres clasificados como osteoporóticos y como no Osteoporóticos.

\begin{tabular}{lccc}
\hline & Nivel 1 & $\begin{array}{c}\text { Hombres clasificados } \\
\text { como Osteoporóticos } \\
(\mathrm{n}=71)\end{array}$ & $\begin{array}{c}\text { Hombres } \\
\text { clasificados como } \\
\text { no osteoporóticos } \\
(\mathrm{n}=118)\end{array}$ \\
\hline Movilidad* n (\%) & Nivel 2 & $30(56.3)$ & $93(78.8)$ \\
& Nivel 3 & $1(1.4)$ & $25(21.2)$ \\
Cuidado Personal n (\%) & Nivel 1 & $64(90.1)$ & $0(0)$ \\
& Nivel 2 & $5(7.0)$ & $113(95.8)$ \\
& Nivel 3 & $2(2.8)$ & $5(4.2)$ \\
Actividades Cotidianas* n (\%) & Nivel 1 & $52(73.2)$ & $0(0)$ \\
& Nivel 2 & $17(23.9)$ & $108(91.5)$ \\
& Nivel 3 & $2(2.8)$ & $10(8.5)$ \\
Dolor/Malestar n (\%) & Nivel 1 & $33(46.5)$ & $0(0)$ \\
& Nivel 2 & $37(52.1)$ & $68(57.6)$ \\
& Nivel 3 & $1(1.4)$ & $44(37.3)$ \\
Ansiedad/Depresión n (\%) & Nivel 1 & $97(82.2)$ & $6(5.1)$ \\
& Nivel 2 & $20(16.9)$ & $97(82.2)$ \\
& Nivel 3 & $1(0.8)$ & $20(16.9)$ \\
& & & $1(0.8)$ \\
\hline
\end{tabular}

Un $42.25 \%$ de los hombres clasificados como osteoporóticos afirma que tiene algún problema para caminar, porcentaje que se reduce a la mitad $(21.19 \%)$ para los hombres que no padecían la enfermedad. Un 56.34\% de las mujeres clasificadas con osteroporosis dice no presentar dificultades para caminar, mientras que un $48.02 \%$ dicen tener algunos problemas en este sentido.

Un $90.14 \%$ de los hombres clasificados como osteoporóticos y un $77.53 \%$ de las mujeres dicen no tener problemas para el cuidado personal. Del grupo de las mujeres clasificadas como osteoporóticas un 18.94\% tienen dificultad para realizar el cuidado personal, en los hombres el porcentaje se reduce a un $7.04 \%$.

Para el grupo de los hombres, un $23.94 \%$ de los pacientes clasificados como osteoporóticos dice tener problemas para realizar las actividades cotidianas (2.82\% para el grupo de las mujeres) y un $2.82 \%$ (6.71\% para las mujeres) son incapaces de realizarlas.

El $42.86 \%$ de los hombres dice tener dolor o malestar moderado (52.93\% para las mujeres) y un $3.70 \%$ (19.28\% para las mujeres) mucho dolor o malestar. Como cabría esperar, presentan mayores niveles de dolor/malestar las mujeres clasificadas como osteoporóticas (59.47\%) que las 
clasificadas como no osteoporóticas $(48.02 \%)$. Resultados semejantes se producen en el grupo de los hombres $(52.11 \%$ frente al $37.29 \%)$.

Si bien el 14.09\% de las pacientes clasificadas como osteoporóticas dice estar muy ansiosa/deprimida, en los hombres clasificados como osteoporóticos este porcentaje se reduce al 1.41\%.

Escala Visual Analógica (EVA). De las 550 mujeres encuestadas, contestaron la escala visual analógica 543 y los 191 hombres encuestados, contestaron a esta pregunta.

La puntuación media dada por las mujeres fue de 62.68 con una desviación típica de 18.02 y la de los hombres de 69.96 con una desviación típica de 16.19. Siendo estas puntuaciones de 61.01 (18.09) para las mujeres y de 68.56 (15.77) para el grupo de los hombres clasificados como osteoporóticos.

Para las comparaciones de los sujetos clasificados como osteoporóticos y de los que no padecen osteoporosis, en los hombres no se han encontrado diferencias significativas en la puntuación media $(p=0.32)$. Sin embargo en el grupo de las mujeres sí se han encontrado diferencias estadísticas significativas en la puntuación media entre los que padecen y no padecen osteoporosis.

Evaluación de la calidad de vida con el cuestionario QUALEFFO

En la Tabla 3 se muestran las medias y los errores estándar para los pacientes osteoporóticos y los no osteoporóticos, en las distintas dimensiones del cuestionario QUALEFFO, para el grupo de los hombres y de las mujeres.

En la Figura 2 se ponen de manifiesto diferencias en las puntaciones medias con valores altos en el grupo de los osteoporóticos, para todas y cada una de las dimensiones del cuestionario QUALEFFO.

Para la dimensión Dolor (D) del cuestionario QUALEFFO debe mencionarse que los valores medios son más bajos para las pacientes clasificadas como no osteoporóticas que para las osteoporóticas, esto no ocurre para el grupo de los hombres. Las diferencias encontradas en las puntuaciones para el grupo de las mujeres son estadísticamente significativas $(\mathrm{p}<0.0001)$.

La dimensión Actividades Cotidianas (AC), como puede observarse en la Figura 2, evidencia diferencias estadísticamente significativas $(p=0.000)$ para el grupo de las mujeres y las puntuaciones medias son más altas en los pacientes (tanto el grupo de los hombres como el grupo de las mujeres) clasificados como osteoporóticos. Con el instrumento genérico para valorar la calidad de vida EUROQOL, también se encontraron dichas diferencias. 
Tabla 3.

Media, desviación estándar y significación del contraste $t$ de Student para las distintas dimensiones del QUALEFFO en pacientes clasificados como osteoporóticos y como no osteoporóticos para el grupo de los hombres y el grupo de las mujeres.

\begin{tabular}{|c|c|c|c|}
\hline & & $\begin{array}{c}\text { Mujeres } \\
(\mathrm{n}=529)\end{array}$ & $\begin{array}{l}\text { Hombres } \\
(\mathrm{n}=189)\end{array}$ \\
\hline \multirow[t]{3}{*}{ Dolor media (DE) } & Osteoporóticos & $2.5(0.1)$ & $1.4(0.1)$ \\
\hline & No Osteoporóticos & $2.1(0.0)$ & $1.5(0.1)$ \\
\hline & $\mathrm{p}$ & 0.000 & 0.847 \\
\hline \multirow[t]{3}{*}{ Actividades Cotidianas media (DE) } & Osteoporóticos & $1.7(0.1)$ & $1.3(0.1)$ \\
\hline & No Osteoporóticos & $1.4(0.0)$ & $1.2(0.0)$ \\
\hline & $\mathrm{p}$ & 0.000 & 0.228 \\
\hline \multirow[t]{3}{*}{ Tareas Domésticas media (DE) } & Osteoporóticos & $2.2(0.1)$ & $1.4(0.1)$ \\
\hline & No Osteoporóticos & $1.6(0.0)$ & $1.2(0.0)$ \\
\hline & $\mathrm{p}$ & 0.000 & 0.034 \\
\hline \multirow[t]{3}{*}{ Movilidad media (DE) } & Osteoporóticos & $2.3(0.1)$ & $1.6(0.1)$ \\
\hline & No Osteoporóticos & $1.7(0.0)$ & $1.4(0.0)$ \\
\hline & $\mathrm{p}$ & 0.000 & 0.029 \\
\hline \multirow[t]{3}{*}{ Actividades Sociales media (DE) } & Osteoporóticos & $1.7(0.1)$ & $1.0(0.0)$ \\
\hline & No Osteoporóticos & $1.1(0.0)$ & $1.0(0.0)$ \\
\hline & $\mathrm{p}$ & 0.000 & 0.274 \\
\hline \multirow[t]{3}{*}{ Estado de Ánimo media (DE) } & Osteoporóticos & $3.5(0.1)$ & $2.8(0.1)$ \\
\hline & No Osteoporóticos & $2.9(0.1)$ & $2.6(0.1)$ \\
\hline & $\mathrm{p}$ & 0.000 & 0.262 \\
\hline \multirow[t]{3}{*}{ Salud General media (DE) } & Osteoporóticos & $2.9(0.1)$ & $1.7(0.1)$ \\
\hline & No Osteoporóticos & $2.3(0.1)$ & $1.7(0.1)$ \\
\hline & $\mathrm{p}$ & 0.000 & 0.682 \\
\hline
\end{tabular}

Lo mismo sucede para las dimensiones Actividades Sociales y de Tiempo libre (AS), Estado de Ánimo (EA) y Percepción de la Salud General $(\mathrm{SG})$. Hay diferencias altamente significativas $(\mathrm{p}=0.000)$, apareciendo un claro gradiente de las clasificadas como no osteoporóticas a osteoporóticas.

En las dimensiones del cuestionario QUALEFFO Actividades Cotidianas (AC) y Tareas Domésticas (TD) presentan diferencias estadísticas altamente significativas $(\mathrm{p}=0.000)$, tanto para el grupo de los hombres como para el grupo de las mujeres, alcanzando las puntuaciones medias más altas los pacientes clasificados como osteoporóticos. La evaluación con el EuroQol-5D, puso también de manifiestó estas diferencias. 


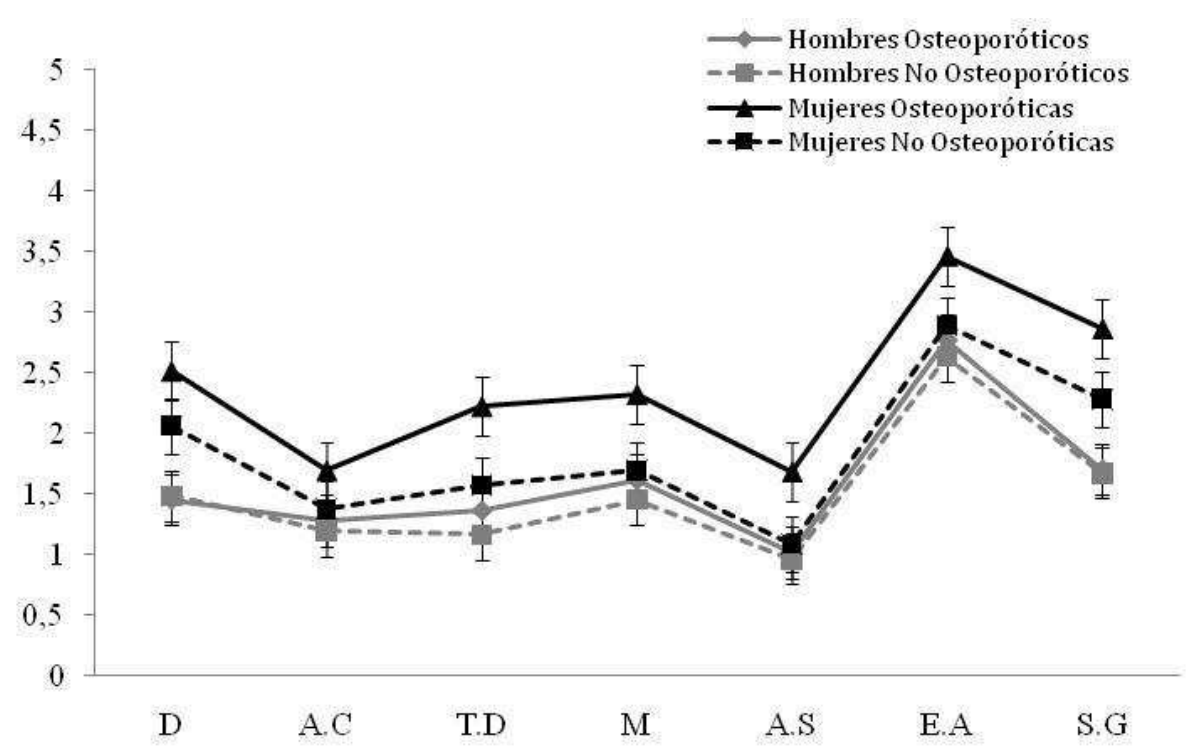

Figura 2. Valores medios y errores estándar para las distintas dimensiones del QUALEFFO en pacientes clasificados como osteoporóticos y como no osteoporóticos para el grupo de los hombres y el grupo de las mujeres.

Nota. a D: Dolor; AC: Actividades Cotidianas; TD: Tareas Domésticas; M: Movilidad; AS: Actividades Sociales; EA: Estado de Ánimo; SG: Salud General.

\section{Discusión}

$\mathrm{Al}$ aplicar en nuestro estudio el instrumento EuroQol-5D, comprobamos que puede ser aplicable para determinar la CVRS, para padecimientos crónicos-degenerativos como la osteoporosis en las mujeres, la cual por los resultados obtenidos tiene repercusiones en todas las dimensiones. Esto no es así en el grupo de los hombres ya que tan solo hemos encontrado diferencias significativas entre pacientes clasificados como osteoporóticos y no osteoporóticos en las dimensiones Movilidad y Actividades Cotidianas.

Como cualquier instrumento de CVRS, el EQ-5D presenta desventajas además de ventajas. Una de ella es la existencia de un efecto techo (ceiling effect) relativamente importante en el sistema descriptivo del EQ-5D. Esto significa que una parte importante de estas poblaciones puntúan el máximo (11111) en esta parte del instrumento. Este hallazgo es por otra parte normal, dado que es esperable que en muestras representativas de la población general la mayor parte de los individuos tengan una buena salud. En nuestro 
estudio la mayor parte, tanto de los hombres como de las mujeres, manifestó no tener problemas en las dimensiones de Movilidad, Cuidado personal, Actividades Cotidianas y Ansiedad/Depresión. En cuanto a la dimensión del Dolor/Malestar, se encontró que más de la mitad de las mujeres de la muestra presentaban dolor moderado y sin embargo porcentaje similar era el que se presentaba en el grupo de los hombres que no presentaban Dolor/Malestar. Un 33.27\% de las mujeres dicen presentar algún problema de ansiedad/depresión, lo que coincide con otros autores (Lydick, Allison \& Barbara, 1996), de que la osteoporosis causa afectación de la calidad de vida en la dimensión de presentar temor.

Cuando se revisó la EVA, comprobamos que los resultados obtenidos para el grupo de las mujeres no son coincidentes con los de otros autores (Tirso, Ramírez, Díez \& Orduña, 2001), ya que un 65\% de la muestra dieron puntuaciones superiores a $70 \mathrm{y}$ en nuestro estudio estas puntuaciones solo las dieron un $43.28 \%$ de las mujeres.

Pero en el caso que nos ocupa, la osteoporosis, al igual que otros autores (Tirso, et al, 2001; Chandler, Martín, Girman, Ross, Love-MacClung, Lydick \& Yawn, 1998) consideramos que los cuestionarios genéricos se encuentran sesgados al no poner de relieve limitaciones funcionales que afectan de manera clara la calidad de vida. Asimismo coincidimos con otras investigaciones (Dolan et al, 1999; Salked, Cameron, Cumming, Easter, Seymour, Kurrle \& Quine, 2000) que al evaluar la calidad de vida mediante diversos instrumentos aplicados en padecimientos musculoesqueléticos, se pudo establecer que la CVRS de las personas afectadas en este sistema, se ve alterada en las diversas dimensiones, dependiendo del problema de que se trate.

$\mathrm{Al}$ administrar el cuestionario QUALEFFO en Atención primaria hemos obtenido que las puntuaciones dadas tanto para el grupo de los hombres como para el grupo de las mujeres fueron mayores para los pacientes clasificados como osteoporóticos que para los clasificados como no osteoporóticos. Estas diferencias son significativas para el grupo de las mujeres en todas las dimensiones del cuestionario QUALEFFO y para el grupo de los hombres solo las dimensiones Tareas Domésticas y Movilidad; luego el cuestionario también ha resultado útil para evaluar calidad de vida en Atención primaria.

No existen referencias que hayan aplicado el cuestionario QUALEFFO en Atención primaria, probablemente por la gran carga asistencial que soportan los médicos y el tiempo que requiere su utilización (20 minutos aproximadamente). Además, algunos profesionales no siempre confían en la validez de la información proporcionada por estos cuestionarios, y otros consideran que el tiempo que lleva su aplicación incrementa la ansiedad del profesional que se siente sobrecargado por su labor asistencial, por lo que se resisten a su aplicación (Lizán, 1998). 
No obstante, progresivamente se va extendiendo el uso de estos y otros cuestionarios para valorar la CVRS y no solo con fines de investigación. En una gran parte de pacientes en atención primaria y especializada, se tiene una idea remota de cómo una patología concreta afecta al estado de salud y la vida cotidiana y esta visión es la que nos pueden ofrecer estos instrumentos (Lizán \& Badía, 2003).

\section{Referencias}

Alegre, L. \& Nualart, J. (1995). Influencia de las variables sociodemográficas y de la salud en la valoración de los estados de salud del EuroQol por pacientes crónicos. Comunicación presentada al VI Congreso de la SESPAS. Gaceta Sanitaria.

Alonso, J. (2002). La calidad de vida relacionada con la salud en la investigación y la práctica clínica: La medida de la calidad de vida relacionada con la salud. Barcelona: Institut Municipal dÍnvestigacions Mèdiques (IMIM).

Abidanza, M. (2005). Recomendaciones generales sobre la osteoporosis. Manejo de la Osteoporosis en Atención primaria. Madrid: Sociedad Española de Medicina Rural y Generalista y Scientific Comunication Management, S.L.

Badía, X. \& Hermand, M. (1999). Adaptación transcultural al español de los cuestionarios OQLQ y QUALEFFO para la evaluación de la calidad de vida relacionada con la salud en mujeres con fractura vertebral osteoporótica. Revista Española de Enfermedades Metabólicas Óseas, 8, 136-140.

Chandler, J. M., Martin, A. R, Girman, C., Ross, P. D., Love-McClung, B., Lydick, E. \& Yawn, B. (1998). Reliability of an Osteoporosis-Targeted Quality of Life Survey Instrument for use in the community: OPTQoL. Osteoporosis International, 8(2), 127-35.

Consensus development conference: diagnosis, prophylaxis, and treatment of osteoporosis. (1993). American Journal of Medicine, 94(6), 646-650.

Díaz, M. (2006). Actualizaciones en Osteoporosis para médicos de Atención primaria. Madrid. Laboratorios Alter. Ed. Ergon.

Dolan, P., Torgerson, D. \& Kakarlapudi, T. K. (1999). Health related quality of life of Colles'fracture patients. Osteoporosis International, 9, 196-199.

Frost, M., Blake,G. \& Fogelman, I. (2000). Can the WHO criteria for diagnosing osteoporosis be applied to calcaneal quantitative ultrasound? Osteoporosis Internationa, 11, 321-330.

García, E., García, E., Mateo, L. \& Holgado, S. (2008). Osteoporosis del varón. Reumatología, 742.

He, Y., Fan, B., Hans, D., Li, J., Wu, C, \& Njeh, F. (2000). Assessment of a new quantitative ultrasound calcaneus measurement; precision and discrimination of hip fractures in elderly women compared with Dual X-Ray Absorptiometry. Osteoporosis International, 11, 354-369.

International Osteoporosis Foundation. Osteoporosis ans you. Recuperado de: www.osteofound.org/publications/pdf/osteoporosis_and_you.pdf [last accessed 8 July 2006].

Gómez de Tejada, M. J. \& Sosa, M. (2002). Los ultrasonidos, la desintometría, el T-score y los criterios de la OMS para el diagnóstico de la osteoporosis. Revista Española de Enfermedades Metabólicas Oseas, 11, 165-166.

Lips, P., Cooper, C., Agnus, D., Caulin, F., Johnell, O. \& Kanis, J. (1999). Quality of life with vertebral fractures: validation of the Quality of Life Questionnaire of the European Foundation for Osteoporosis (QUALEFFO). Osteoporosis International, 10, 150-60. 
Lizán Tudela, L. (1998). Viabilidad de las viñetas COOP/WONCA y otros instrumentos para la valoración de la calidad de vida relacionada con la salud en atención primaria. Atención primaria, 22, 135-9.

Lizán Tudela L. \& Badia, X. (2003). La evaluación de la calidad de vida en la osteoporosis. Cuestionarios en Atención primaria, 31(2), 126-133.

Lydick, E., Allison, M. \& Barbara, Y. (1996). Impact of fears on quality of life in patients with a silent disease: Osteoporosis. Clinical Therapeutics, 18(6), 1307-1315.

Olesksik, A., Lips, P., Dawson, A., Minshall, M.E., Shen, W., Cooper, C. \& Kanis, J. (2000). Health related quality of life in postmenopausal women with low BMD with or without prevalent vertebral fractures. Journal of Bone and Mineral Research, 15(7), 1384-1392.

Orozco, P., Montero, J. C., Prieto, D. (2009). Diagnostico de la osteoporosis en Atención primaria. En Orozco, P. (Ed.) Pautas de actuación en osteoporosis para Atención primaria (pp. 7-20). Barcelona: Profármaco.2.

Ortega, A. (2005). Diagnóstico de la osteoporosis. En Abidanza, G. M., Ortega, C. A. y Moya, S. R. (Ed.) Manejo de la Osteoporosis en Atención primaria (pp. 41-76). Madrid, D.F.: Sociedad Española de Medicina Rural y Generalista y Scientific Comunication Management, S.L.

Randell, A. G., Nguyen, T. V., Bhalerao, N., Silverman, S. L., Sambrook, P. N. \& Eisman, J. A. (2000). Deterioration in quality of life following hip fracture: A prospective study. Osteoporosis International, 11(5), 460-466.

Rué, M. \& Badia, X. (1996). The Spanish EuroQol Tariff: Results from the Catalan Health Survey based on self-rated health. En Badía, X.; Herdman, M.; Segura, A. (Ed.) EuroQol Plenary Meeting 1995 Discussion Papers (pp. 77-98). Barcelona: Institut Universitari de Salut Publica de Catalunya.

Salked, G., Cameron, I. D., Cumming, R. G., Easter, S., Seymour, J., Kurrle, S. E. \& Quine, S. (2000). Quality of life related to fear of falling and hip fracture in older women: a time trade of study. British Medical Journal, 320 (7231), 341-6.

Velardo-Jurado, E.; Ávila-Figueroa, M.C. (2002). Consideraciones metodológicas para evaluar Calidad de Vida. Salud Pública de Mexico, 44(5), 448-463.

Sánchez, 2008. Aportaciones al Análisis de Datos de Calidad de Vida Relacionada con la Salud, desde una Perspectiva Multivariante. Tesis Doctoral: Universidad de Salamanca.

Sosa, M. \& Gómez de Tejada, M. J. (2006). Densitometría vs ultrasonidos en el diagnóstico de la osteoporosis. En Díaz, M (Ed.) Actuaciones en Osteoporosis para médicos de Atención primaria (pp. 5-12). Madrid: ERGON.

Sosa, M. \& Gómez de Tejada, M. J. (2008). Epidemiogía de la osteoporosis en el varón. En Díaz, M (Ed.) Actuaciones en Osteoporosis masculina para médicos de Atención primaria (pp. 512). Madrid: ERGON.

Tirzo, A., Ramírez, E., Diez, P., \& Orduña, A. (2001). Evaluación de la calidad de vida en personas con osteoporosis tratadas en el Centro Nacional de Rehabilitación-Ortopedia (CNR-O). Revista Mexicana de Medicina Física y Rebabilitación, 13, 14-20.

Recibido: 22 de enero de 2010 Aceptado: 14 de enero de 2011 